\title{
O brincar como recurso terapêutico ocupacional no tratamento de crianças com Paralisia Cerebral
}

\author{
The playing as an occupational therapeutic resource in the treatment of children with \\ Cerebral Palsy
}

El jugar como recurso terapéutico ocupacional para el tratamiento de niños con Parálisis Cerebral

Ana Clara Fragallo Ferreira ${ }^{1 *}$, Emilly Oliveira Sales ${ }^{1}$, Ana Paula Corrêa Ribeiro ${ }^{1}$, Flávio Roberto Pereira Tavares ${ }^{1}$, Karina Saunders Montenegro'.

\section{RESUMO}

Objetivo: Analisar a produção científica dos terapeutas ocupacionais no Brasil sobre os benefícios do brincar como recurso no tratamento de crianças com Paralisia Cerebral (PC). Métodos: Revisão bibliográfica integrativa, de abordagem quanti-qualitativa, do tipo descritivo e exploratório, para adquirir conhecimento a respeito da ludicidade como recurso terapêutico para crianças com PC. Resultados: Observa-se que todos os artigos encontrados foram estudos de caso, nos quais foram identificados benefícios do brincar para crianças com PC, dentre eles o aperfeiçoamento de capacidades motoras, a possibilidade de relações sociais e de uma forma de expressão. Considerações finais: Compreende-se o brincar como um vantajoso objetivo terapêutico e recurso para a minimização dos efeitos da PC, o qual apresenta efeitos positivos no processo de aprendizagem e no aperfeiçoamento de habilidades motoras, de raciocínio e informações sensoriais. Também destaca-se um baixo número de artigos sobre esta temática, tendo em vista sua importância, e logo, a necessidade de novos estudos.

Palavras-chave: Terapia ocupacional, Brincadeiras, Paralisia cerebral.

\begin{abstract}
Objective: Analyze the scientific production of brazilians occupational therapists on the benefits of playing as a resource in the treatment of children with Cerebral Palsy (CP). Methods: Integrative bibliographic review, a quanti qualitative approachment, of the descriptive and explanatory kind, in order to acquire knowledge about the ludicity as a therapeutic resource for children with CP. Results: It is noted that all the articles found were case studies, in which benefits of playing for children with CP have been identified, including the improvement of motor skills, the possibility of social relationships and a way of expression. Final considerations: It is concluded that playing is a beneficial therapeutic objective and a resource for the minimization of CP effects, wich has positive effects on the learning process and improving motor skills, reasoning and sensory information. Likewise, the low number of articles on this subject stands out, considering its importance, thus, further research are necessary.
\end{abstract}

Key words: Occupational theraphy, Play and playthings, Cerebral palsy.

\section{RESUMEN}

Objetivo: Analizar la producción científica de terapeutas ocupacionales en Brasil sobre los beneficios del juego como recurso en el tratamiento de niños con parálisis cerebral. Métodos: Revisión bibliográfica

${ }^{1}$ Universidade do Estado do Pará (UEPA), Belém - PA. *E-mail: fragalloana@gmail.com 
integradora, con abordaje cuantitativo y cualitativo de manera descriptiva y exploratoria, para adquirir conocimientos sobre los juegos como recurso terapéutico para niños con PC. Resultados: Es posible observar que todos los artículos encontrados fueron estudios de caso, en los quales se fueron identificados los beneficios del juego para niños con PC, entre ellos la mejora en la motricidad, la posibilidad de relaciones sociales y las maneras expresión. Consideraciones finales: Se entiende el juego como un objetivo terapéutico ventajoso y como recurso para minimizar los efectos de la PC, en el cual presenta efectos positivos en el proceso de aprendizaje, en la mejora de la motricidad, en el razonamiento y en la información sensorial. También cabe mencionar el escaso número de artículos sobre este tema, dada su importancia y, por tanto, la necesidad de realizar más estudios.

Palabras clave: Terapia ocupacional, Juegos, Parálisis cerebral.

\section{INTRODUÇÃO}

A Paralisia Cerebral (PC) também conhecida como Encefalopatia Crônica Não Progressiva na Infância (ECNI), decorre de uma lesão no encéfalo em desenvolvimento, na qual compromete as funções motoras e a postura, apresentando alterações que afetam diretamente o desenvolvimento da criança acometida. Estas crianças podem também apresentar alterações sensoriais, funcionais e cognitivas, assim como alterações do tônus muscular e padrões anormais de postura, comunicação e percepção (SILVA CS e LACERDA RAMV, 2017; DIAS ACB, et al., 2010; ZILLI F, 2013).

A paralisia cerebral pode ser classificada de duas formas: classificação anatômica, quando se considera as partes do corpo que são afetadas, e classificação clínica, em que se analisa os sintomas do paciente, podendo manifestar-se em várias áreas do corpo. A classificação anatômica divide-se em diparética, quando afeta os membros inferiores, hemiparética, na qual apenas um lado do corpo é afetado e tetraparética, onde tronco e membros são comprometidos (FERNANDES AC, et al., 2007).

Em relação à classificação clínica pode ser espática, quando ocorre lesão no sistema piramidal e caracteriza-se pela presença de movimentos serpenteados, espasmos musculares e movimentos que levam a distúrbios posturais. Atáxica, que acomete o cerebelo e suas vias, refletindo em movimentos descoordenados e dismetria. Discinética, decorre da lesão dos núcleos de base que se reflete em movimentos involuntários que somem durante o sono, hipotonia axial e distúrbios na fala (FERNANDES AC, et al., 2007).

O trabalho de intervenção em paralisia cerebral deve ser realizado por equipe multiprofissional, para assim promover o desenvolvimento e a aquisição de habilidades funcionais, além de corrigir e prevenir deformidades. Neste contexto de intervenção deve-se destacar a importância do trabalho do terapeuta ocupacional contribuindo com esta equipe. É de responsabilidade do terapeuta ocupacional avaliar o desempenho psicomotor $\mathrm{e}$ as habilidades manuais, também desenvolver as capacidades motoras e perceptivas do paciente/cliente, além de avaliar e tratar possíveis alterações no processamento sensorial, realizando sempre atividades e tarefas que favoreçam a autonomia e independência da criança, não esquecendo de possibilitar uma melhor interação em atividades escolares e atividades da vida diária (AVD'S) (ZILLI F, 2013 apud LOWES LP e GREIS SM, 2002).

Além do que já foi exposto, ressalta-se também que o terapeuta ocupacional tem um papel fundamental na área de prescrição e adaptação de cadeiras de rodas e na confecção de órteses que visam o posicionamento adequado, além de buscar manter ou aumentar a amplitude de movimento dos membros superiores ou inferiores. O terapeuta ocupacional irá avaliar a demanda de cada criança, o atendimento precisa ser individualizado e assim elencar entre os vários métodos e abordagens terapêuticas que podem ser aplicados durante o processo de intervenção de uma criança com PC. Este profissional deverá sempre buscar critérios rigorosos para suprir as necessidades específicas de cada criança (ZILLI F, 2013).

Durante as intervenções do terapeuta ocupacional o brincar é considerado uma atividade essencial, compondo elementos sensoriais, motores, cognitivos, afetivos e sociais. Assim, o brincar em crianças com PC irá favorecer a eles ganhos motores e contribuir para o processo de reabilitação (PERES LW, et al., 2018). 
Além disso, os recursos lúdicos e as motivações utilizadas são mecanismos que despertam nas crianças vontade de realizar as atividades propostas, tornando a atividade mais prazerosa e auxiliando a enfrentar as barreiras apresentadas pela doença (BRAGA MAM e GRACIANI Z, 2015; SANTOS TR, et al., 2011).

Portanto, esta revisão bibliográfica integrativa objetivou analisar a produção científica dos terapeutas ocupacionais no Brasil sobre os benefícios do brincar como recurso no tratamento de crianças com PC, para responder à questão norteadora: "Quais os benefícios identificados por terapeutas ocupacionais ao analisar o brincar como recurso no tratamento de crianças com paralisia cerebral?".

\section{MÉTODOS}

Este estudo trata-se de uma revisão bibliográfica integrativa, de abordagem quanti-qualitativa, do tipo descritivo e exploratório. Mendes KDS, et al. (2008) afirmam que na revisão integrativa busca-se, através da análise de documentos científicos, o preenchimento de lacunas sobre determinado assunto, utilizando teorias pré-estabelecidas na criação de novas hipóteses e elucidação de perguntas sem respostas.

Mendes KDS, et al (2008), também afirmam que a revisão integrativa divide-se em 6 etapas: (1) criação do tema e da questão norteadora, (2) criação do critérios de exclusão e inclusão para a pesquisa e seleção de material, (3) definição das informações relevantes e que devem ser extraídas dos artigos, (4) avaliação dos estudos que foram incluídos na tentativa de solucionar possíveis conflitos entre artigos, (5) interpretação dos resultados e (6) apresentação dos conhecimentos que foram sintetizados através da interpretação (Figura 1).

Figura 1 - Fluxograma de pré-análise dos artigos.

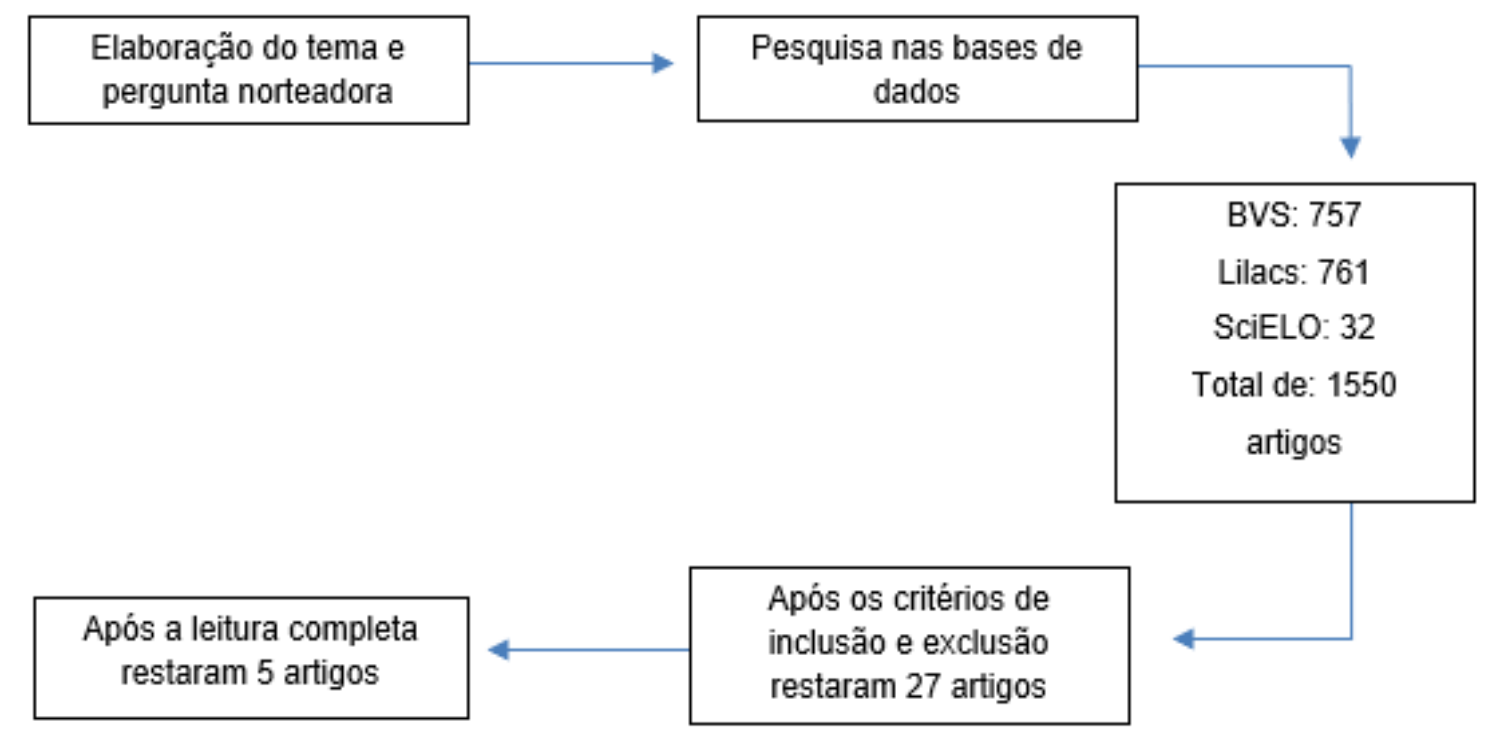

Fonte: Ferreira ACF, et al., 2021.

As buscas foram realizadas nas bases de dados eletrônicas Biblioteca Virtual em Saúde (BVS), Literatura Latino-americana e do Caribe em Ciências da Saúde (LILACS) e Livraria Cientifica Eletrônica Online (SciELO). Foram utilizados nas buscas os descritores: terapia ocupacional, paralisia cerebral, brincar (brinc) e os operadores lógicos "AND" e "OR" para combinação dos descritores utilizados na seleção das publicações. A coleta dos dados ocorreu no período entre 28 de outubro a 22 de novembro de 2020 .

Foram critérios de inclusão: (1) artigos em português, (2) completos, (3) gratuitos, (4) publicados nos últimos 10 anos (2010-2020), (5) que tivessem entre os autores no mínimo um terapeuta ocupacional, (6) relacionados a crianças com paralisia cerebral, e (7) contendo no mínimo dois dos três descritores: brincar (brinc* ${ }^{*}$ atividade lúdica), terapia ocupacional (terapeuta ocupacional) e paralisia cerebral (encefalopatia 
crônica não evolutiva da infância) no título, no resumo e/ou nas palavras-chaves. Os critérios de exclusão foram: (1) Trabalhos referentes a resumos de anais em eventos científicos e (2) Cartas ao editor.

Os dados quantitativos foram registrados em ocorrência e frequência, e organizados em tabelas. Analisouse o perfil dos artigos quanto ao ano de publicação, local de publicação, caracterização dos estudos quanto aos métodos utilizados (estudos de revisão bibliográfica, estudos de campo, estudos de caso, ensaios clínicos etc.) e quanto ao perfil dos autores dos artigos.

A análise qualitativa ocorreu a partir da leitura exaustiva dos artigos, e os resultados foram organizados em três categorias de análise: Benefícios do brincar, Dificuldades/barreiras enfrentadas pelas crianças com paralisia cerebral e Intervenções do Terapeuta Ocupacional. Espera-se que através da discussão destas categorias seja possível responder à questão norteadora deste estudo: Quais os benefícios identificados por terapeutas ocupacionais ao analisar o brincar como recurso no tratamento de crianças com paralisia cerebral?

\section{RESULTADOS E DISCUSSÃO}

No que se refere aos dados quantitativos, foram organizados de acordo com o ano de publicação, local de publicação, tipos e abordagens de pesquisa utilizadas e perfil dos autores dos artigos. Durante a pesquisa foi encontrado um total de 1550 artigos (Tabela 1).

Tabela 1 - Total de artigos encontrados.

\begin{tabular}{lccc}
\hline \multicolumn{1}{c}{ Descritores/combinações } & BVS & Lilacs & Scielo \\
\hline Terapia ocupacional AND paralisia cerebral & 405 & 405 & 10 \\
\hline Terapia ocupacional AND brinc & 232 & 95 & 15 \\
\hline Paralisia cerebral AND brinc & 100 & 261 & 6 \\
\hline Terapia ocupacional AND paralisia cerebral AND brinc & 20 & 0 & 1 \\
\hline
\end{tabular}

Fonte: Ferreira ACF, et al., 2021.

Após a aplicação dos critérios de inclusão e exclusão foram excluídos 3 artigos que se repetiam em diferentes plataformas de pesquisa. Após a leitura completa foi excluído um artigo que não tinha pertinência com o tema, restando somente 5 artigos, os quais serão discutidos adiante (Tabela 2).

Tabela 2 - Estudos encontrados após a aplicação dos critérios de inclusão e exclusão.

\begin{tabular}{lccc}
\multicolumn{1}{c}{ Descritores/combinações } & BVS & Lilacs & Scielo \\
\hline Terapia ocupacional AND paralisia cerebral & 1 & 1 & 1 \\
\hline Terapia ocupacional AND brinc & 1 & 0 & 1 \\
\hline Paralisia cerebral AND brinc & 1 & 1 & 0 \\
\hline Terapia ocupacional AND paralisia cerebral AND brinc & 1 & 0 & 0 \\
\hline Total de artigos & & $\mathbf{5}$ & \\
\hline
\end{tabular}

Fonte: Ferreira ACF, et al., 2021.

A análise dos dados quantitativos iniciou-se através do registro da ocorrência e frequência quanto ao ano de publicação. Identificou-se que $20 \%$ dos artigos foram produzidos no ano de $2010,20 \%$ no ano de 2011 , $20 \%$ no ano de 2012, 20\% no ano de 2016, 20\% no ano de 2017. Entre os anos de 2013 e 2015 não houve a publicação de nenhum artigo relacionado ao tema. Além disso, do ano de 2018 em diante não houve novas publicações referentes ao tema no Brasil.

Zanini G, et al., (2009), relata que o número estimado de ocorrência de paralisia cerebral varia entre 30 a 40 mil novos casos por ano no Brasil. Contudo, observa-se que o elevado número de casos não reflete o número de estudos sobre o tema. Portanto, deve haver maior incentivo a pesquisas sobre o tema, pois através dela encontram-se novos métodos de tratamento além do desenvolvimento de intervenções mais eficientes no campo da Terapia Ocupacional (Tabela 3). 
Tabela 3 - Referente ao ano de publicação.

\begin{tabular}{ccc}
\hline Ano de publicação & N & $\%$ \\
\hline 2010 & 1 & 20 \\
2011 & 1 & 20 \\
2012 & 1 & 20 \\
2016 & 1 & 20 \\
1017 & 1 & 20 \\
\hline
\end{tabular}

Fonte: Ferreira ACF, et al., 2021.

Com relação a região de publicação nota-se maior ocorrência na região sudeste estando distribuídos entre São Paulo com $80 \%$ das publicações, e Minas Gerais com $20 \%$ (Tabela 4).

Tabela 4 - Referente ao local de publicação.

\begin{tabular}{ccc}
\hline Local de publicação & N & $\%$ \\
\hline São Paulo & 4 & 80 \\
\hline Minas Gerais & 1 & 20 \\
\hline
\end{tabular}

Fonte: Ferreira ACF, et al., 2021.

Percebe-se que por ser polo de circulação de informações a cidade de São Paulo tem maior número de publicações referentes ao tema, acredita-se que este Estado apresente um maior número de investimentos a pesquisa científica, seja da área pública ou privada, como consequência de seu destaque dentro do país por ser uma das maiores cidades.

Montenegro KS, et al. (2020) reforçam que, levando em consideração a magnitude do nosso país, devese considerar que existe uma variação significativa a nível cultural, regional e social. Assim, também seria importante o desenvolvimento de mais estudos, em diferentes contextos, regiões e culturas.

No que se refere ao tipo de abordagem de pesquisa, $40 \%$ foram de pesquisas qualitativas e $60 \%$ de abordagem quanti-qualitativa, não houve nenhuma pesquisa de caráter apenas qualitativo. Quanto ao tipo de pesquisa observou-se que todos eram pesquisas de campo, variando entre relato de caso, relato de pesquisa e estudos descritivos.

Com relação a ausência de pesquisas com abordagem apenas quantitativa, justifica-se pelo fato de a Terapia Ocupacional ser muito humanizada, não apenas voltada para os resultados ou números, mas sim para uma abordagem holística considerando o contexto, realidade e dando voz aos pacientes, caracterizando uma abordagem mais focada no lado qualitativo.

Sobre a formação acadêmica dos autores dos artigos, notou-se que houve grande prevalência de terapeutas ocupacionais $70 \%$, enquanto que $15 \%$ eram enfermeiros, $5 \%$ pediatras, $5 \%$ médicos fisiatras e $5 \%$ biólogos. Também se identificou que $95 \%$ dos autores atuavam na área da saúde enquanto os 5\% restantes foram ocupados por ciências biológicas, com $95 \%$ dos autores formados em universidades pública e $5 \%$ em instituições privadas (Tabela 5).

Tabela 5 - Referente a formação acadêmica.

\begin{tabular}{ccc}
\hline Formação acadêmica & N & $\%$ \\
\hline Terapeuta Ocupacional & 15 & 70 \\
\hline Enfermeiros & 3 & 15 \\
\hline Medica fisiatra & 1 & 5 \\
\hline Pediatra & 1 & 5 \\
\hline Biólogo & 1 & 5
\end{tabular}

Fonte: Ferreira ACF, et al., 2021.

Observou-se que por ser um assunto que está dentro das competências da Terapia Ocupacional houve grande número de pesquisadores terapeutas ocupacionais, no mais foi evidenciado a presença de outros 
profissionais, o que ressalta a importância da interdisciplinaridade na construção do conhecimento cientifico. Um dos objetivos de intervenção, na prática clínica da Terapia Ocupacional, é o desempenho e independência de crianças com PC, em contextos relevantes, na realização de tarefas de vida diária (GUERZONI VPD, et al., 2008).

O estudo de Gama BTB, et al. (2020) reforça que os terapeutas ocupacionais precisam se dedicar mais na publicação de suas intervenções, que a comunidade tenha ferramentas para compreender melhor o processo de tratamento de sua criança, principalmente estudos com pesquisa de campo e estudos de caso que abordem os benefícios do tratamento. Torna-se necessário assim identificar os objetivos de cada artigo e verificar a aproximação com os objetivos desta pesquisa.

O artigo A1 aponta a necessidade dos profissionais que acompanham as crianças com deficiência física colaborarem com os pais para a efetivação e a ampliação do repertório do brincar de seus filhos, contribuindo para a participação social e a autonomia da criança (SOUZA DS, et al., 2017)

$\mathrm{O}$ artigo A2 caracteriza-se como um relato de experiência de atuação de acadêmicas em terapia ocupacional com uma criança com PC, discutindo os principais aspectos abordados durante o período de intervenção e os desfechos clínicos que favoreceram o desenvolvimento e a qualidade de vida do paciente, assim como, a influência da participação do cuidador como suporte e forma de incentivo, transmitindo as experiências sensório motoras e funcionais dos atendimentos para as atividades do seu cotidiano (BARCELOS TA, et al., 2012).

O artigo A3 objetivou demonstrar que os instrumentos Avaliação do Comportamento Lúdico e a Entrevista Inicial com os Pais são capazes de identificar o comportamento lúdico de crianças com paralisia cerebral e também possibilitam visualizar com maior clareza os interesses e as capacidades lúdicas da criança avaliada, permitindo, assim, traçar metas funcionais e delinear o plano terapêutico (SILVA CS, et al. 2016).

$\mathrm{O}$ artigo A4 trata-se de um estudo de campo, o qual buscou demonstrar através de entrevista inicial com os pais conhecer o comportamento lúdico da criança em casa por meio de oito perguntas, as quais compreendiam: o interesse da criança, seu modo de comunicar, os brinquedos conhecidos e utilizados, algumas características de seu brincar, seus parceiros de brincadeira, bem como sua atitude lúdica e a avaliação do comportamento lúdico (ZAGUINI CGS, et al., 2011).

Findando, o artigo A5 teve como objetivo utilizar brinquedos como recurso terapêutico no alívio das tensões reais e inconscientes da criança em relação à hospitalização, utilizando os próprios materiais hospitalares como: luvas, tocas, aventais etc., para familiarizar a criança com os instrumentos hospitalares e assim reduzir as tensões do pré-operatório (FONTES CMB, et al., 2010).

Observa-se que apesar de os artigos encontrados abordarem os temas principais sobre terapia ocupacional, paralisia cerebral e o brincar, e embora todos falem sobre os benefícios do brincar para o desenvolvimento da criança com PC, estes benefícios não foram o foco principal dos estudos. Esperava-se encontrar estes resultados principalmente porque todas as pesquisas foram de campo.

Quanto ao perfil dos artigos evidenciou-se não haver prevalência de artigos publicados em um determinado ano. Referente ao local de publicação, 100\% das publicações ocorreram na região sudeste. Também se identificou que $95 \%$ dos autores atuavam na área da saúde, sendo $70 \%$ terapeutas ocupacionais. Além disso, observou-se que $60 \%$ dos artigos utilizaram abordagem quanti-qualitativa, e que todos os artigos eram pesquisas de campo.

Quanto a análise dos dados qualitativos, buscou-se responder à pergunta norteadora, por meio do qual constatou-se que os terapeutas ocupacionais identificaram benefícios do brincar para crianças com PC, como o aperfeiçoamento de suas capacidades motoras e informações sensoriais, assim como a possibilidade de relações sociais. O brincar é identificado, também, como uma forma de expressão e criatividade, favorecendo o desenvolvimento de habilidades físicas e cognitivas, além do desenvolvimento interpessoal e alívio de tensões. Ademais, o brincar fornece à criança um domínio sobre o seu próprio corpo e sobre o ambiente, construindo sua identidade e tornando-a um ser social. 
Para melhor entendimento os resultados coletados foram organizados em três categorias de análise: benefícios do brincar, dificuldades/barreiras enfrentadas pelas crianças com paralisia cerebral, e intervenção do Terapeuta Ocupacional.

\section{Benefícios do brincar}

Todos os artigos relataram que o brincar traz benefícios à criança com paralisia cerebral, no seu desenvolvimento motor, cognitivo, social, afetivo e/ou cultural. Através da expressão e criatividade que a ocupação proporciona, é fornecido um meio de construção da identidade do paciente, além de despertar interesses e atitudes similares às crianças sem deficiência, o que pode garantir a ele uma sensação de normalidade. Para isso, devem ser estimuladas brincadeiras de forma espontânea e livre, pois assim a atividade é feita com atitude, trazendo o benefício da autonomia.

Para a criança com deficiência, o brincar é de extrema importância para o desenvolvimento de suas habilidades motoras, por meio da manipulação de objetos de diferentes texturas e tamanhos, além de atividades que podem facilitar sua movimentação e controle postural, como relatado no artigo 2, oferecendo um desempenho motor mais coordenado (BARCELOS TA, et al, 2012).

Foi destacado também o benefício da interação social, como foi identificado pelos autores nos artigos 3 e 5 , uma vez que o brincar pode facilitar o contato com outras crianças e objetos, e possibilitar a exploração e exteriorização de seus sentimentos, favorecendo, assim, seu desenvolvimento e crescimento (FERLAND F, 2006 apud SILVA CMA, et al., 2016). Além disso, o brincar apresentou-se como um instrumento de comunicação entre o paciente e o profissional, já que a atividade lúdica, através da dramatização, pode contribuir para um seguro esclarecimento da condição da criança e dos procedimentos realizados, diminuindo a sensação de medo.

Identificou-se ainda que o brincar para a criança com PC é um mecanismo de aprendizagem, pois permite a exploração do seu corpo e do ambiente, auxilia nas suas habilidades de raciocínio e aperfeiçoa as informações sensoriais.

\section{Dificuldades/barreiras enfrentadas pelas crianças com paralisia cerebral.}

Sabe-se que a principal característica da PC é o déficit motor, entretanto essas desordens motoras são frequentemente acompanhadas por alterações da sensação, da cognição, da comunicação, da percepção e/ou do comportamento, e/ou por crises convulsivas (BRASIL, 2013; CRUZ DMC e PFEIFER LI, 2006; GIANNI MA, 2003 apud SILVA CMA, 2016).

Estas alterações podem representar um curso particular no desenvolvimento e crianças com PC podem apresentar pouca vivência de experiências sensório-motoras, restrição na exploração e manipulação dos objetos, o que pode levar a uma limitação da vivência nas brincadeiras em seu cotidiano (BRASIL, 2013; CRUZ DMC e PFEIFER LI, 2006; GIANNI MA, 2003 apud SILVA CMA, 2016).

Além das dificuldades relacionadas ao quadro clínico e funcional, a criança com deficiência física também enfrenta fatores ambientais (físicos e sociais) que podem comprometer o brincar (MISSUANA C e POLLOCK N, 1991 apud SOUZA DS, et al., 2017).

Os fatores ambientais podem ser tão ou até mais limitadores do que o próprio distúrbio. As barreiras sociais, que ocorrem como resultado da imposição dos valores e crenças dos outros, impossibilitam as crianças de estabelecerem interações sociais. Por outro lado, as barreiras físicas do ambiente dificultam a locomoção, bem como diminuem ou impedem o acesso das crianças à recreação e ao lazer (LORENZINI MV, 2002 apud SOUZA DS, et al., 2017; ROCHA, et al., 2018; BALTOR MRR e DUPAS G, 2013).

A aparência física diferente, os movimentos do corpo não usuais e a comunicação verbal, expressa por sons incompreensíveis, são exemplos de características que podem desviar ou absorver toda a atenção do outro para aquilo que é o não esperado e, explicitamente, diferente, não restando olhar para outros atributos que as crianças com deficiência possam ter. Às vezes, não raras, os atributos relativos ao estigma parecem se manifestar em outras características que nem são da pessoa (TAKATORI M, et al., 2001 apud SOUZA DS, 2017). 
Ademais, foi identificado que devido às lesões do sistema nervoso central (SNC), juntamente com disfunções no sistema musculo esquelético, as crianças com PC apresentam atrasos no desenvolvimento de habilidades motoras, e por isso são necessárias intervenções voltadas para a funcionalidade motora (BARCELOS TA, et al., 2012).

Assim, a criança com PC pode apresentar dificuldades em diversas atividades, incluindo o brincar por diversos fatores como: barreiras no acesso ao brinquedo, dificuldades no manuseio do mesmo, relações interpessoais e condições ambientais.

\section{Intervenção do Terapeuta Ocupacional}

O profissional da terapia ocupacional possui um papel muito importante, uma vez que este intervém com objetivos de minimizar os efeitos de déficits, tendo como instrumento as atividades, enquanto busca meios alternativos para alcançar metas funcionais específicas, de acordo com as características pessoais, dificuldades e habilidades apresentadas pelo paciente durante o atendimento (BARCELOS TA, et al., 2012).

Neste sentido, para o tratamento de crianças com PC podem ser realizadas intervenções com o brincar como recurso terapêutico primordial, sendo este um meio de motivação para a criança durante todo o processo, uma vez que esta é uma atividade inerente ao comportamento infantil.

$O$ ato de brincar deve ser facilitado, para que a criança possa explorar, exteriorizar seus sentimentos, por meio desta experiência lúdica. É necessário dar-lhes oportunidades de participação nas brincadeiras, facilitando o contato com outras crianças, adultos e objetos, favorecendo, com isso, o desenvolvimento e crescimento da mesma, seja através de brinquedos normativos ou terapêuticos (ZAGUINI CGS, et al., 2011).

Para a estimulação neuromotora, pode-se realizar manuseios, facilitando a movimentação da criança. É possível utilizar-se de bolas suíças, rolos ou até o colo do próprio terapeuta para o desempenho mais coordenado da movimentação requerida (BARCELOS TA, et al., 2012). De igual modo, como recurso para estimulação sensorial, pode ser oferecidos à criança brinquedos de diferentes formas, texturas, pesos, tamanhos, sons e cores.

Ademais, faz-se necessário que a família compreenda a importância do brincar para o desenvolvimento da criança com PC, tendo em vista que os parentes mais próximos, geralmente os pais, são os principais parceiros de brincadeiras e que as crianças, poucas vezes, iniciam uma brincadeira com independência, dependendo da sensibilidade dos pais para perceberem que elas querem brincar (TAKATA N, 1974 apud SOUZA DS, et al., 2017).

Compreende-se, então, após a leitura exaustiva dos artigos, que as crianças com PC podem ser beneficiadas ao realizarem a ocupação do brincar, melhorando seu desempenho motor, possibilitando interações sociais e uma alternativa de mecanismo de aprendizagem. Como essa deficiência apresenta algumas desordens a nível funcional e podem apresentar dificuldades na realização das atividades, seja no acesso ou manuseio dos brinquedos, seja em relações interpessoais, a atuação da terapia ocupacional tornase fundamental, por meio de intervenções subjetivas e individuais que ofereçam oportunidades e motivação aos clientes.

\section{CONSIDERAÇÕES FINAIS}

O brincar apresenta efeitos positivos em crianças com Paralisia Cerebral, que vão desde o processo de aprendizagem, ao desenvolvimento de relações sociais, bem como o aperfeiçoamento de habilidades motoras, de raciocínio e informações sensoriais, ressaltando que estas brincadeiras devem ser estimuladas de forma espontânea e livre, para que, igualmente, haja o benefício da autonomia. A intervenção terapêutica ocupacional compreende o brincar não apenas como um recurso para a minimização dos efeitos da Paralisia Cerebral, mas também como objetivo terapêutico, tendo em vista que, devido às dificuldades relacionadas ao quadro clínico e funcional, a criança enfrenta limitações que comprometem sua vivência nas brincadeiras em seu cotidiano. Destaca-se como limitação deste estudo o baixo número de artigos encontrados sobre esta temática, e logo, a necessidade do desenvolvimento de novos estudos. 


\section{AGRADECIMENTOS}

Agradecimentos à Karem Raquel Lessa Farnum, formada em Letras Espanhol pela Universidade Federal do Pará (UFPA), Castanhal-Pará, por realizar a tradução do português para o espanhol referente ao resumo deste artigo.

\section{REFERÊNCIAS}

1. BALTOR MRR, DUPAS G. Experiences from families of children with cerebral paralysis in context of social vulnerability. Rev. Latino-Am. Enfermagem, 2013; 21(4): 956-963.

2. BARCELOS TA, et al. A atuação da terapia ocupacional em hospital pediátrico. Rev. Med. Minas Gerais, 2012; 22: 88-91.

3. BRAGA MAM, GRACIANI Z. O brincar na rotina da criança com paralisia cerebral. Cadernos de Pós-Graduação em Distúrbios do Desenvolvimento, 2015; 15(1): 41-49.

4. DIAS ACB, et al. Desempenho funcional de crianças com paralisia cerebral participantes de tratamento multidisciplinar. Fisioterapia e Pesquisa, 2010; 17(3): 225-9.

5. FERNANDES AC, et al. AACD Medicina e Reabilitação: Princípios e prática. São Paulo: Artes Médicas, 2007; 976p.

6. FONTES CMB, et al. Utilização do brinquedo terapêutico na assistência à criança hospitalizada. Rev. Bras. Ed. Esp., 2010; 16(1): 96-106.

7. GAMA BTB, et al. Seletividade alimentar em crianças com Transtorno do Espectro Autista (TEA): uma revisão narrativa da literatura. Revista Artigos.com, 2020; 17: 1-11.

8. GUERZONI VPD, et al. Análise das intervenções de terapia ocupacional no desempenho das atividades de vida diária em crianças com paralisia cerebral: uma revisão sistemática da literatura. Rev. Bras. Saúde Mater. Infant., 2008; 8(1): 17-25.

9. MENDES KDS, et al. Revisão integrativa: método de pesquisa para a incorporação de evidências na saúde e na enfermagem. Texto contexto - enferm., 2008; 17(4): 758-764.

10. MONTENEGRO KS, et al. Desempenho ocupacional de cuidadores de crianças com transtorno do espectro autista (tea): uma revisão da literatura. Revista Eletrônica Acervo Saúde, 2020; (56), e4033.

11. PERES LW, et al. Estratégias lúdicas na reabilitação motora de crianças com paralisia cerebral: revisão integrativa. Revista Eletrônica de Enfermagem, 2018; 20.

12. PETERS $\mathrm{C}$, et al. An integrative review of assessments used in occupational therapy interventions for children with cerebral palsy. Cad. Bras. Ter. Ocup., 2019; 27(1): 168-185.

13. ROCHA ANDC, et al. Avaliação da Acessibilidade do Parque Durante o Brincar de Crianças com Paralisia Cerebral na Escola. Rev. bras. educ. espec., 2018; 24(1): 73-88.

14. SANTOS TR, et al. Avaliação do comportamento lúdico de crianças com paralisia cerebral. Arquivos brasileiros de paralisia cerebral, $2011 ; 5(11): 18-25$.

15. SILVA CMA, et al. Percepção de Pais e Terapeutas Ocupacionais sobre o Brincar da Criança com Paralisia Cerebral. Revista Brasileira de Educação Especial, 2016; 22(2): 221-232.

16. SILVA CS, LACERDA RAMV. Efeitos do protocolo pedia suit no tratamento de crianças com paralisia cerebral. Revista Multidisciplinar do Nordeste Mineiro, 2017; 192-207.

17. SOUZA DS, et al. O brincar de crianças com deficiência física sob a perspectiva dos pais. Cadernos Brasileiros de Terapia Ocupacional, 2017; 25(2): 267-274.

18. ZAGUINI CGS, et al. Avaliação do comportamento lúdico da criança com paralisia cerebral e da percepção de seus cuidadores. Acta Fisiátrica, 2011; 18(4): 187-191.

19. ZANINI G, et al. Paralisia Cerebral: causas e prevalências. Fisioterapia em Movimento, 2017; 22(3).

20. ZILLI F. Revisão sistêmica dos procedimentos da terapia ocupacional na paralisia cerebral. Revista Baiana de Terapia Ocupacional, 2013; 2(1): 17-28. 